\title{
QCBED-DFT: Experimentally constrained density functional theory
}

\author{
D. Peng ${ }^{1}$, P.N.H. Nakashima ${ }^{2}$ \\ ${ }^{1}$ Department of Physics, Norwegian University of Science and Technology (NTNU), Trondheim 7491, Norway; \\ ${ }^{2}$ Department of Materials Science and Engineering, Monash University, Victoria 3800, Australia,
}

\section{Ding.Peng@ntnu.no}

Quantitative convergent-beam electron diffraction (QCBED) has become renowned for its accuracy and precision when it comes to measuring bonding electrostatic potentials and electron densities [1 - 3]. Density functional theory (DFT) needs no introduction because of its ubiquity in materials science and crystallography. It is efficient but compromised in accuracy by the approximations needed to make it less computationally expensive than many-body wave-function calculations. It is also feared by some that DFT is becoming over-parametrised in the bid to deal with the shortcomings of approximations and is therefore "straying from the path toward the exact functional" [4].

We have integrated DFT into QCBED in such a way that allows DFT model parameters, including parameters associated with density functionals, to be refined by fitting DFT-calculated convergent-beam electron diffraction (CBED) patterns to experimental CBED patterns from a real material. We call this $Q C B E D-D F T$ [5] and illustrate the basic principle of the method in Fig. 1 below.

We will present a number of experimental measurements of density functional parameters such as the Hubbard energy, $U$, in some strongly correlated electron materials, $\mathrm{NiO}$ and $\mathrm{CeB}_{6}$, from our recently published work [5], as well as some new, unpublished trials.

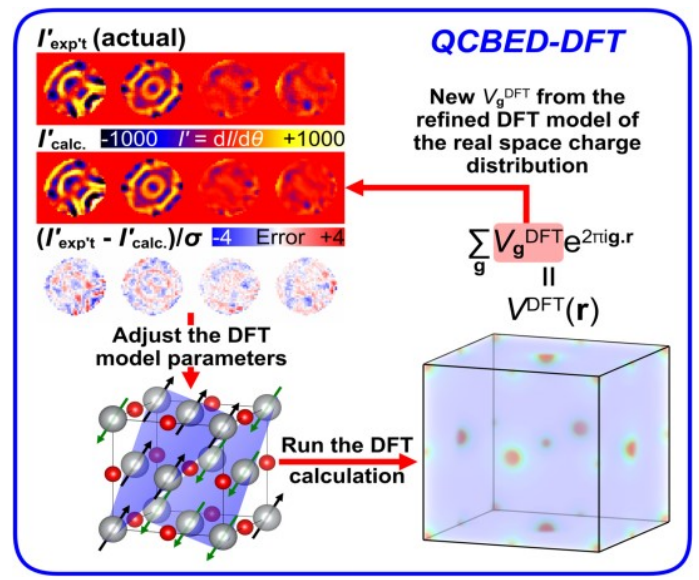

Figure 1. The basic operation of $Q C B E D-D F T$. An experimental CBED pattern from a real material is fitted with a calculated pattern by adjusting the DFT model parameters. This changes the real space electron distribution calculated by DFT, changing all of the structure factors to new values, $V_{\mathbf{g}}$ DFT , instead of a small subset of them as is normally the case in conventional QCBED. These are then used in the calculation of the corresponding simulated CBED pattern that is matched to the experimental one. This figure has been modified from a component of a figure presented in [5].

[1] Zuo, J. -M., Kim, M., O’Keeffe, M. \& Spence, J. C. H. (1999). Nature 401, 49.

[2] Nakashima, P. N. H., Smith, A. E., Etheridge, J. \& Muddle, B. C. (2011). Science 331, 1583.

[3] A. Genoni, L. Bučinský, N. Claiser, J. Contreras-García, B. Dittrich, P.M. Dominiak, E. Espinosa, C. Gatti, P. Giannozzi, J. Gillet, D. Jayatilaka, P. Macchi, A.Ø. Madsen, L. Massa, C.F. Matta, K.M. Merz Jr, P.N.H. Nakashima, H. Ott, U. Ryde, K. Schwarz, M. Sierka, S. Grabowsky (2018). Chem. Eur. J. 24, 10881.

[4] Medvedev, M. G., Bushmarinov, I. S., Sun, J., Perdew, J. P. \& Lyssenko, K. A. (2017). Science 355, 49.

[5] Peng, D. \& Nakashima, P. N. H. (2021). Phys. Rev. Lett. 126, in press.

Keywords: density functional theory; electron diffraction; quantum crystallography; charge densities; chemical bonding

We thank the late Prof. Andrew Johnson. We are grateful to the Monash Centre for Electron Microscopy where the CBED data were collected. Many thanks to Prof. Joanne Etheridge for her valuable advice throughout this work. PN thanks the Australian Research Council for funding (FT110100427 \& DP210100308).

Acta Cryst. (2021), A77, C237 